\title{
307 - Exploratory Study on Healthcare Decision-Making Capacity Assessment
}

Ana Saraiva Amaral ${ }^{1}$; Rosa Marina Afonso ${ }^{2}$; Mário R. Simões ${ }^{3}$; Sandra Freitas ${ }^{4}$

${ }^{1}$ Center for Research in Neuropsychology and Cognitive and Behavioral Intervention (CINEICC), University of Coimbra; Health Sciences Research Centre (CICS-UBI), University of Beira Interior

${ }^{2}$ Faculty of Human and Social Sciences, University of Beira Interior; Center for Health Technology and Services Research (CINTESIS), University of Porto.

${ }^{3}$ Faculty of Psychology and Educational Sciences, University of Coimbra; Center for Research in Neuropsychology and Cognitive and Behavioral Intervention (CINEICC), Assessment and Ageing Processes (NAAP); Psychological Assessment and Psychometrics Laboratory (PsyAssessmentLab), University of Coimbra.

${ }^{4}$ Center for Research in Neuropsychology and Cognitive and Behavioral Intervention (CINEICC), Assessment and Ageing Processes (NAAP); Psychological Assessment and Psychometrics Laboratory (PsyAssessmentLab), University of Coimbra.

Mild cognitive impairment (MCl) and Alzheimer's Disease (AD) prevalence is expected to continue to increase, due to the population ageing. $\mathrm{MCl}$ and $A D$ may impact patients' decision-making capacities, which should be assessed through the disease course. These medical conditions can affect the various areas of decision-making capacity in different ways. Decision-making capacity in healthcare is particularly relevant among this population. Elders often suffer from multimorbidity and are frequently asked to make healthcare decisions, which can vary from consenting a routine diagnostic procedure to decide receiving highly risk treatments.

To assess this capacity in elders with $\mathrm{MCl}$ or $\mathrm{AD}$, we developed the Healthcare DecisionMaking Capacity Assessment Instrument (IACTD-CS - Instrumento de Avaliação da Capacidade de Tomada de Decisão em Cuidados de Saúde). This project is funded by Portuguese national funding agency for science, research and technology, FCT (SFRH/BD/139344/2018). IACTD-CS was developed based on Appelbaum and Grisso four abilities model, literature review and review of international assessment instruments.

After IACTD-CS first version development, an exploratory study with focus groups was conducted. This study included focus groups with healthcare professionals and nursing homes' professionals.

The focus groups main goals were: 1) understand the participants perception regarding healthcare decision-making capacity, 2) distinguish relevant aspects of decision-making, 3) discuss the abilities and items included in IACTD-CS and 4) identify new aspects or items to be added to IACTD-CS. A content analysis of the focus groups results, with resource to MAXQDA, was conducted afterwards. This exploratory study allowed to identify professionals' perceptions on healthcare decision-making and its results were a significant contribute to IACTD-CS development. The proposed communication aims to describe the methodology used and present the results of content analysis. 